\title{
Comparison of Recording Properties of ME tape and Thin MP tape with respect to Overwrite Behavior
}

\author{
S. Lalbahadoersing, J.P.J. Groenland, 'S.B. Luitjens, J.C. Lodder \\ University of Twente, MESA Research Institute, Information Storage Technology Group, \\ P.O.Box 217, 7500 AE Enschede, The Netherlands \\ * Philips Research Laboratories, Professor Holstlaan 4, 5656 AA Eindhoven, The Netherlands
}

\begin{abstract}
Differences between the recording characteristics of thin MP and ME tape are studied. The effect of the thickness reduction of MP tape is also investigated. When thin MP tape (with thickness $140 \mathrm{~nm}$ ) is compared with ME tape (with thickness $150 \mathrm{~nm}$ ), we observe a better signal and overwrite response for the ME tape. Through simulations the influence of an easy axis out-of-plane and a different reversal mechanism in ME tape is related to overwrite behavior.
\end{abstract}

\section{INTRODUCTION}

To meet the requirements in the DV system [1] there is an ongoing competition between MP and ME tape. Due to the double-coating technology (introduced by Fuji [2]) MP tape can produce an output level comparable with ME tape. Another point of interest is the overwrite behavior. It has been reported [3] that the reduction in thickness in MP tape improves overwrite characteristics. The main source of the overwrite phenomenon seems to be modulation of the new pattern by the old pattern. This modulation is shown [4] to give an overwrite ratio (OWR), which is linked with the hard transition shift $(\Delta)$. The overwrite ratio is defined as [5]

$$
O W R=20 \log \frac{A_{2}}{A_{1}}=20 \log \left(\frac{\Delta}{2 B}\right)
$$

Here, $A_{1}$ is the amplitude of a low-frequency $\left(f_{1}\right)$ pattern, $A_{2}$ is the residual $\left(f_{i}\right)$ amplitude when a high-frequency $\left(f_{2}\right)$ pattern overwrites the $f_{1}$ pattern, $B$ is the bit length of the new signal. This hard transition shift is proportional to the medium demagnetization $\left(H_{d}\right)[6]$ :

$\Delta=\frac{H_{d}}{d H_{\text {heud }} / d x}=\frac{M_{r} \delta(d+\delta / 2)}{H_{c} \pi Q r} F(d, g, r)$

in which $M_{r}$ is the remanent magnetization, $\delta$ is the medium thickness, $d$ is the head-medium spacing, $H_{c}$ is the coercivity, $Q$ is the maximum head field gradient $(-0.8), r$ is the length of the writing zone and $F$ is included to take into

Manuscript received January 28, 1997.

S. Lalbahadoersing, Phone X-31-53-4894434, Fax X-31-53-4893343, s.lalbahadoersing @el.utwente.nl,

http://www.el.utwente.nl/tdm/istg/istghome.htm.

The financial support for the running project is provided by the Dutch Technology Foundation STW account imaging. It may be noticed that the transition length $(a$ $\left.=\sqrt{ } M_{r} \delta\left(d+\frac{1}{2}\right) /\left(\pi Q H_{c}\right)\right)$ scales with the hard transition shift (e.g. [6]). When the transition length is affected by a change in the medium parameters, this will also change the hard transition shifts and thus to first order the overwrite behavior. It is therefore interesting to know whether an introduction of an easy axis out-of-plane and a different reversal mechanism (giving a simulated MP tape ME tape-like characteristics), change the transition length and thus the overwrite behavior. This is studied by simulations.

\section{EXPERIMENTAL}

Three experimental MP tapes with small advanced MP particles are studied to look at the effects of thickness reduction. The comparison is valid because other tape parameters are kept constant. These are: $M_{r} \approx 320 \mathrm{kA} / \mathrm{m}$, $H_{c}=185 \mathrm{kA} / \mathrm{m}$, squareness $S \approx 0.9$, switching field distribution $S F D \approx 0.28$, orientation factor $O F \approx 2.4$ and roughness $R_{a} \approx 4 \mathrm{~nm}$. One $\mathrm{MP}$ tape $\left(\mathrm{MP}_{1.3}\right)$ has a magnetic coating with thickness $\delta \approx 1.3 \mu \mathrm{m}$, the second MP tape $\left(\mathrm{MP}_{0.23}\right)$ has a $\delta \approx 0.23 \mu \mathrm{m}$ and the third sample $\left(\mathrm{MP}_{0.14}\right)$ has a $\delta \approx 0.14 \mu \mathrm{m}$. The ME tape used for comparison is a commercially available tape for the DV system. The data for this tape are: $M_{r} \approx 610 \mathrm{kA} / \mathrm{m}, H_{c} \approx 120 \mathrm{kA} / \mathrm{m}$ and $\delta \approx 0.150$ $\mu \mathrm{m}$ [7] with an easy axis angle out-of-plane of $40^{\circ}$ (with the longitudinal direction).

For the recording measurements a rotating tape-drum with an air inlet to optimize the head-medium spacing was used. The rotation velocity of the drum was $1 \mathrm{~m} / \mathrm{s}$. We used a TSS MIG head with a gaplength $g \approx 0.25 \mu \mathrm{m}$, number of turns $N=20$ and a trackwidth $W=25 \mu \mathrm{m}$. The recording current was optimized for the output voltage at a wavelength of $\lambda=1 \mu \mathrm{m}$. The voltage-current characteristic did not show saturation of the head for the applied currents. A spectrum analyzer is used to detect the readback signal.

Two types of measurements are carried out to investigate the overwrite behavior. In the first one the write wavelength $\lambda_{1}$ is varied $(>1.33 \mu \mathrm{m})$ and the overwrite wavelength $\lambda_{2}$ is taken as $1 \mu \mathrm{m}$, comparable with [8]. In the other measurement the current is varied with fixed $\lambda_{1}=2 \mu \mathrm{m}$ and $\lambda_{2}=0.5 \mu \mathrm{m}$ [9]. The currents of the new and the overwritten signal have equal values. Here $\lambda_{2}$ is the shortest wavelength used in the DV system, which employs partial response class IV equalization. 


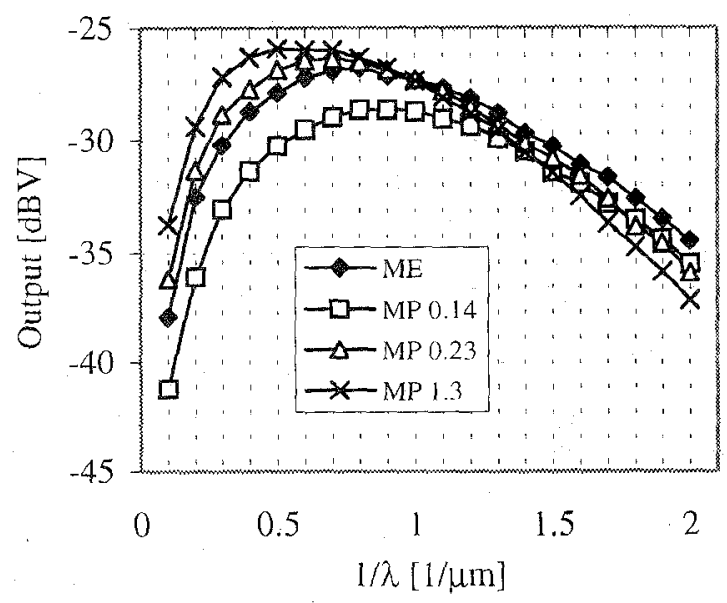

Fig. 1 Measured frequency spectra of four tapes

\section{RESULTS}

The measured frequency spectra (Fig. 1) show that, for $\lambda<1 \mu \mathrm{m}$, the three MP tapes have lower output values than the ME tape. The thickness reduction of the MP tape leads to an increase of the output in the high-frequency region. The gain of $1.7 \mathrm{dBV}$ in output of this $\mathrm{MP}_{0.14}$ tape at $\lambda=0.5 \mu \mathrm{m}$ however, is lost in the large wavelength region (7.4 dBV at $\lambda=10 \mu \mathrm{m})$. We see that the thinnest MP tape does not show here a higher output than the ME tape in the measured frequency region.

The overwrite ratio (OWR) as a function of the inverse wavelength (Fig. 2) shows that the $\mathrm{MP}_{1.3}$ and $\mathrm{MP}_{0.23}$ start with a difference in OWR value of $4 \mathrm{~dB}$ and end with a difference smaller than $0.5 \mathrm{~dB}$, in the given region. A thickness reduction, as in the case of the $\mathrm{MP}_{0.14}$, gives a tremendous improvement of overwrite behavior, with a maximum value of about $12.5 \mathrm{~dB}$. When this $\mathrm{MP}_{0.14}$ is compared with the ME tape it shows at the average $2.8 \mathrm{~dB}$ higher value of the OWR.

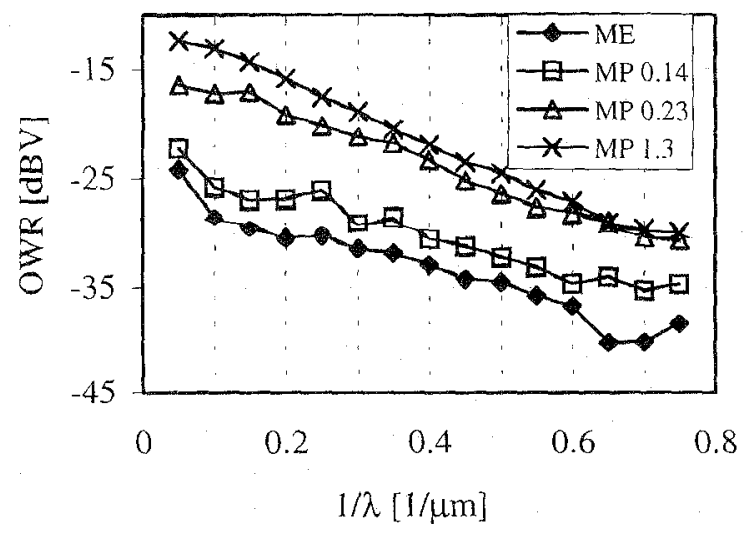

Fig. 2 Measured overwrite ratio (OWR) as a function of inverse wavelength for three MP tapes with different thicknesses and one ME tape.

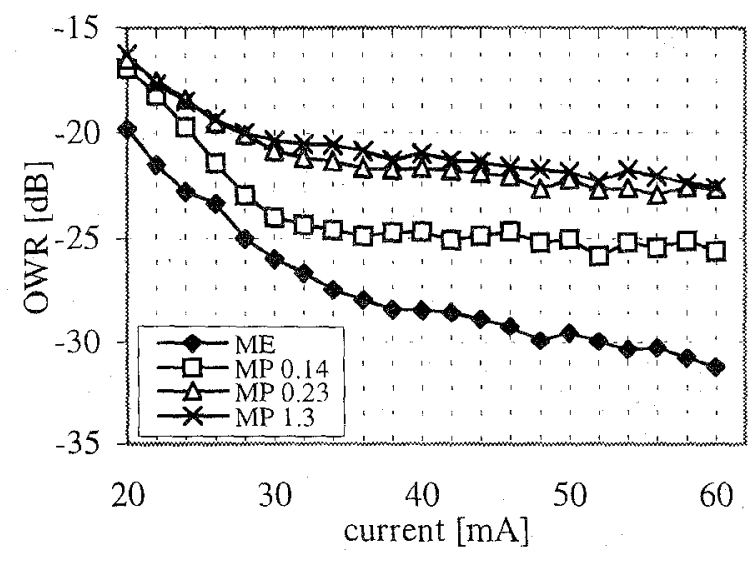

Fig. 3 Measured overwrite ratio as a function of current for three MP tapes with different thicknesses and one ME tape.

The overwrite ratio $(f 2 / f l=4)$ as a function of the current (Fig. 3) shows that the $M P_{1.3}$ and $M P_{0.23}$ can give a $8.6 \mathrm{~dB}$ worse overwrite behavior with respect to $\mathrm{ME}$ tape. When the thickness of the magnetic coating is reduced to $0.14 \mu \mathrm{m}$, an improvement is seen of the overwrite behavior, but even at this thickness value ME tape shows at the average $3.7 \mathrm{~dB}$ better performance.

\section{SIMULATIONS}

It can be seen that although the thicknesses of $\mathrm{MP}_{0.14}$ tape and the ME tape are of the same order of magnitude they differ with respect to signal output and overwrite behavior in favor of ME tape. To investigate this further simulations are made with a self-consistent model, based on a Moving-Preisach Stoner-Wohlfarth vector hysteresis model [10]. In this numerical recording model the transition length $(a)$ can be studied. Its behavior is investigated as a function of easy axis angle out-of-plane (which is typical for ME tape). In the simulation of the $\mathrm{MP}_{0.23}$ tape an estimated head-medium spacing $(d)$ of $50 \mathrm{~nm}$ is assumed. In this simulated tape an easy axis angle out-of-plane is introduced. In Fig. 4 the calculated $\mathrm{PW}_{50}$ as a function of varying angle is given. (The absolute value of the measured $\mathrm{PW}_{50}$ is $470 \mathrm{~nm}$.) The $g, \delta$ and the $d$ values are kept constant in our simulation, so that the only changing parameter in the $P W_{50}$ (given by $P W_{50}=\sqrt{\left[g^{2}\right.}$ $+4(d+a)(d+a+\delta)])$ is the transition length $(a)$. Now it can be concluded from this figure that an increasing out-ofplane angle will decrease the transition length, indicating that the overwrite behavior can improve as an easy axis angle outof-plane is introduced. However, when the output is considered for two different wavelengths namely $1 \mu \mathrm{m}$ and $0.5 \mu \mathrm{m}$ we see that the output changes $2 \mathrm{dBV}$ for $1 \mu \mathrm{m}$ and $<$ $0.5 \mathrm{dBV}$ for $0.5 \mu \mathrm{m}$ at $50^{\circ}$ with respect to the signal output in plane.

MP tape and ME tape may also differ in their reversal mechanisms. For particulate media reversal takes place 


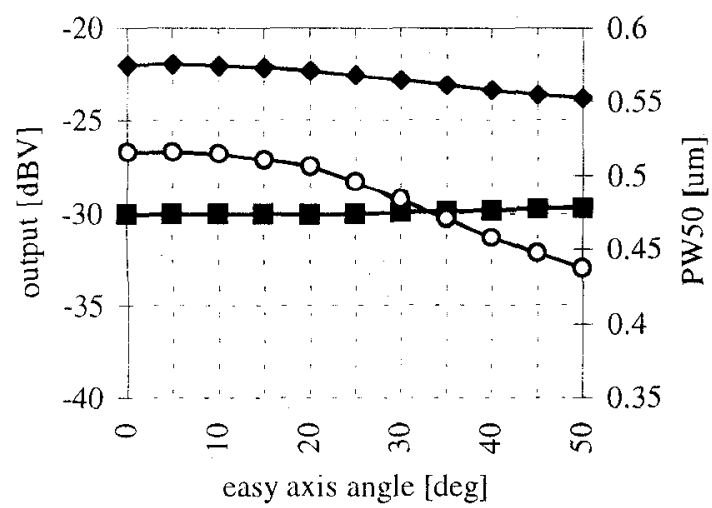

Fig. 4 The computed output for $\lambda=1 \mu \mathrm{m}$ (diamonds) and $\lambda=0.5 \mu \mathrm{m}$ (squares) and the $P W_{50}$ (circles) with respect to easy axis angle out-of-plane.

mainly by incoherent rotation, while thin-film media also display domain-wall-motion type reversals [11]. A measure for this can be $H_{i} / H_{k}[10]$ in which $H_{r}$ is the remanence coercivity and $H_{k}$ is the anisotropy field. The value used for the fit is $H_{1} / H_{k}=0.45$. When we vary this parameter in such a way that we are going gradually to domain-wall-motion type reversals we see that the $\mathrm{PW}_{50}$ decreases, which means smaller transition lengths. The output changes for $\lambda=1 \mu \mathrm{m}$ $\sim 1 \mathrm{dBV}$ and for $\lambda=0.5 \mu \mathrm{m} \sim 2 \mathrm{dBV}$ (see Fig. 5).

\section{CONCLUSION}

Overwrite experiments show that the $\mathrm{MP}_{0.14}$ has a better overwrite behavior than $\mathrm{MP}_{1.3}$ and $\mathrm{MP}_{0.23}$. Thickness reduction gives a better overwrite characteristic because the transition length is also reduced. This could be through the removal of the lower part of the magnetic coating where the transition becomes broader, resulting in a smaller transition

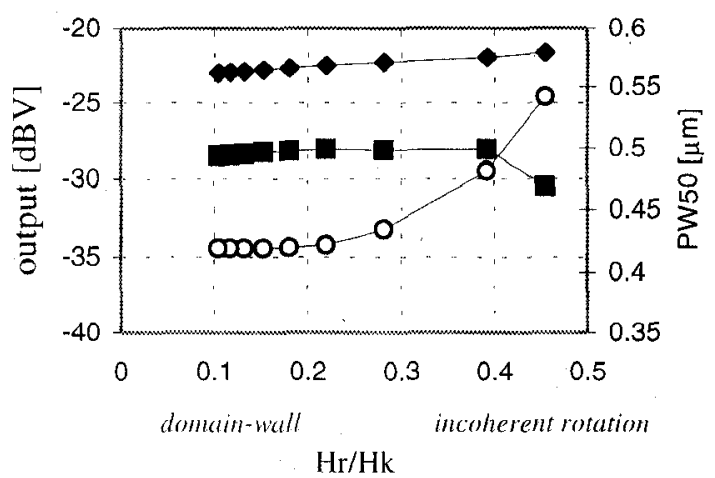

Fig. 5 The computed output for $\lambda=1 \mu \mathrm{m}$ (diamonds) and $\lambda=0.5 \mu \mathrm{m}$ (squares) and the $\mathrm{PW}_{511}$ (circles) with respect to reversal-mechanism. At the horizontal axis we have given the type of reversals which will mainly occur at given values of $H_{i} / H_{k}$ length. A disadvantage which shows to arise is that the thickness reduction also can give an output reduction in the low-frequency region.

$\mathrm{MP}_{0.14}$ however, did not show a better overwrite behavior compared with ME tape, although it has a thickness value of the same order. Overwrite and output properties of the ME tape were found to be superior.

In our simulations MP tape is modeled. In this simulated tape the addition of an easy axis out-of-plane and changes with respect to the reversal mechanism are studied to follow the transformation from MP tape to ME tape-like characteristics. The problem of signal reduction can be handled by adjusting the out-of-plane angle and $H_{r} / H_{k}$ in such a manner that the signal loss becomes negligible.

Differences in easy axis and reversal mechanisms of MP- and $\mathrm{ME}$ tape might explain a narrower $\mathrm{PW}_{50}$ for $\mathrm{ME}$ tape, corresponding with a smaller transition length, resulting in a better overwrite behavior for ME tape.

\section{ACKNOWLEDGMENT}

The authors would like to thank Ing.M. Siekman for his technical assistance. They are also grateful to Dr.P. Heilmann from BASF Magnetics for supplying the MP samples.

\section{REFERENCES}

[1] C. Yamamitsu, A. Iketani, J. Ohta and N. Echigo, "An Experimental Digital VCR for Consumer use", IEEE Trans. Magn., vol..31 , no 2. March 1995, 1037-1043

[2] H. Inaba, K. Ejiri, N. Abe, K. Masaki and H. Araki, "The Advantages of the Thin Magnetic Layer on a Metal Particulate Tape", IEEE Trans. Magn., vol. 29, no 6, November 1993, 3607-3612

[3] H.J. Richter and R.J. Veitch, "MP tape for high density digital recording", Journal of Magnetism and Magnetic Materials 155 (1996), $80-82$

[4] G.H. Lin Y. Zhao and H.N. Bertram, "Overwrite in Thin Film Disk Recording Systems", IEEE Trans. Magn., vol. 29, no 6, November $1993,4215-4223$

[5] J. Fitzpatrick and X. Che., "The dependence of overwrite on proximity effects", IEEE Trans. Magn., vol.32, no 5, September 1996, 3869-3871

[6] H.N. Bertram, Theory of Magnetic Recording, Ch. 9, Cambridge University Press, 1994

[7] S.B. Luitjens, S.E. Stupp and J.C. Lodder, "Metal evaporated tape: state of the art and prospects", J. Magn. Magn. Mater. 155 (1996), 261-265

[8] A. Stek, S.E. Stupp and S.B. Luitjens, "Some Aspects of Overwrite in Thick Magnetic Media", IEEE Trans. Magn., vol. 32, no 5, September 1996, 4055-4057

[9] A. Friedmann, D. Wei, H.N. Bertram, J.K. Wolf, R.Swanson and F.Jeffers, "Experimental analysis on the effects of tape thickness on magnetic recording", J. Appl. Phys. 79 (8), is April 1996, 5658-5660

[10] H.A.J. Cramer, "A Moving Preisach Vector Hysteresis model for magnetic recording media”, J. Magn. Magn. Mater. 88 (1990), 194-204

[11] J.C. Lodder Ph.D. thesis 1995, " Co-Cr-(X) thin films for high density recording", University of Twente, Enschede, The Netherlands 